\title{
Relação da alimentação com surgimento precoce da obesidade e diabetes mellitus tipo 2 em crianças e adolescentes
}

\author{
Relationship of feeding with early emergence of obesity and mellitus type 2 diabetes in children \\ and adolescents \\ Relación de laalimentaciónconsurgimiento precoce de laobesidad y diabetes mellitus tipo 2 en \\ niños y adolescentes
}

Aline Oliveira de Brito Silva ${ }^{1}$, Adolfo Marcito Campos de Oliveira ${ }^{2}$, Carla Maria Pontes da Silva ${ }^{3 *}$, Daiara Dávila Jorge de Oliveira ${ }^{3}$, Daniela Teresa da Silva Carrias ${ }^{3}$, Herivelton de Araújo Rodrigues ${ }^{3}$, Juniel José de Morais $^{4}$, Kassiane Araújo Rodrigues ${ }^{5}$, Lissandra de Sousa Rocha Barros ${ }^{3}$, Moira Raísa Vasconcelos de Souza ${ }^{3}$, Radson Neves de Araújo ${ }^{3}$, Rosane Kelly Silva Ramos ${ }^{6}$, Whyds Rodrigues de Lima.

\section{RESUMO}

Objetivo: Analisar na literatura produções científicas acerca da relação dos hábitos alimentares para o surgimento da obesidade e do diabetes mellitus tipo 2 na infância e adolescência. Metodologia: Pesquisa bibliográfica realizada na Biblioteca Virtual em Saúde: Scientific Electronic Library Online (SciELO), Literatura Latino-americana e do Caribe em Saúde (LILACS) e da Medical Literature Analysis and Retrieval System Online (MEDLINE), do período de 2008 a 2018. Resultados e Discussão: Foram selecionados 10 artigos vinculados à área, onde a literatura mostrou que o consumo das crianças e adolescentes está desequilibrado e essa realidade são fatores precursores do aumento das doenças crônicas como o diabetes mellitus nesta fase precoce que é a adolescência, acarretando ainda problema de saúde coletiva no futuro. Considerações finais: Torna-se necessário alertar a população dos riscos nutricionais que podem surgir durante a infância e adolescência, sendo de grande importância a reeducação alimentar e a implementação de atividades de educação nutricional nas escolas, as quais podem auxiliar na formação de hábitos alimentares saudáveis, na manutenção da saúde e controle de peso corporal adequado.

Palavras-chave: Alimentação, Diabetes Mellitus, Obesidade infantil.

\begin{abstract}
Objective: To analyze in the literature scientific productions about the relationship of eating habits to the appearance of obesity and type 2 diabetes mellitus in childhood and adolescence. Methodology: A bibliographical study carried out in the Virtual Health Library: Scientific Electronic Library Online (SciELO), Latin American and Caribbean Health Literature (LILACS) and the Medical Literature Analysis and Retrieval System Online (MEDLINE), from 2008 to 2018. Results and Discussion: We selected 10 articles related to the area, where the literature showed that the consumption of children and adolescents is unbalanced, and this reality are precursors to the increase of chronic diseases such as diabetes mellitus in this early phase that is adolescence, leading to still a collective health problem in the future. Final considerations: It is necessary to alert the population to the nutritional risks that may arise during childhood and adolescence, and it is of great importance to reeducation and the implementation of nutritional education activities in schools, which can help in the formation of healthy eating habits, in maintaining proper health and body weight control.
\end{abstract}

Key words: Feeding, Diabetes Mellitus, Childhood Obesity.

\footnotetext{
${ }^{1}$ Associação de Ensino Superior - AESPI, Teresina-Piauí.

2 Universidade Federal da Paraíba, Campina Grande-Paraíba.

${ }^{3}$ Centro Universitário Santo Agostinho, Teresina-Piauí. * E-mail: carlapontes07@hotmail.com

${ }^{4}$ Centro Universitário Uninovafapi, Teresina-Piauí.

${ }^{5}$ Faculdade do Piauí - FAPI, Teresina-Piauí.

${ }^{6}$ Faculdade Integral Diferencial - FACID, Teresina-Piauí.

7 Universidade Federal do Piauí - UFPI, Teresina-Piauí.
} 


\section{RESUMEN}

Objetivo: Analizarenla literatura producciones científicas acerca de larelación de los hábitos alimenticios para elsurgimiento de laobesidad y de la diabetes mellitus tipo 2 enlainfancia y adolescencia. Metodología: Investigación bibliográfica realizada enla Biblioteca Virtual en Salud: Scientific Electronic Library Online (SciELO), Literatura Latino americana y del Caribe en Salud (LILACS) y del Medical Literature Analysis and Retrieval System Online (MEDLINE), del período de 2008 a 2018. Resultados y Discusión: Se seleccionaron 10 artículos vinculados al área, donde la literatura mostró que el consumo de los niños y adolescentes está desequilibrado y esa realidad son factores precursores del aumento de las enfermedades crónicas como la diabetes mellitus en esta fase precoz que es la adolescencia, acarreando aún problema de salud colectiva En el futuro. Consideraciones finales: Es necesario alertar a la población de los riesgos nutricionales que pueden surgir durante la infancia y la adolescencia, siendo de gran importancia la reeducación alimentaria y la implementación de actividades de educación nutricional en las escuelas, las cuales pueden auxiliar en la formación de hábitos alimentarios saludables, en el mantenimiento de la salud y control de peso corporal adecuado.

Palavras-Chave: Alimentación, Diabetes Mellitus, Obesidad infantil.

\section{INTRODUÇÃO}

A alimentação é importante e está diretamente relacionada à sobrevivência e ao bem-estar. No Brasil, a Lei Orgânica da Saúde define que um dos fatores determinantes e condicionantes para a saúde é a alimentação, que é componente fundamental dos direitos da criança (BRASIL, 2009).

Sabe-se, que os hábitos alimentares exercem grande influência sobre a saúde, o crescimento e o desenvolvimento dos indivíduos. Neste sentido, é através dos modelos de ingestão que é realizada na infância e adolescência torna-se possível que a obesidade, bem como, as doenças crônicas na fase adulta. Além disso, ressalta-se que alguns tipos de câncer relacionados à alimentação realizada ao longo dos anos (NEUTZLING et al. 2010).

Para Muller et al. (2014) a obesidade infantil e na adolescência vem aumentando progressivamente nas últimas décadas, sendo considerada uma epidemia global pela Organização Mundial da Saúde. Os dados epidemiológicos apresentandos no ano de 2014, apontou existência de 43 milhões de crianças menores de 5 anos com sobrepeso no mundo, dentre essas, 35 milhões em países em desenvolvimento e 8 milhões em países desenvolvidos.

Deve-se destacar que no que tange ao diabetes mellitus, a sua incidência entre crianças e adolescentes torna-se um grande problema de saúde pública. E assim esforços são empreendidos, em vários níveis, tem sido realizado com o objetivo de se detectar fatores responsáveis pela eclosão da doença nessa faixa etária, passíveis de correção ou intervenção. Entretanto, paralelamente ao número cada vez maior de casos, observa-se a incidência crescente de formas da doença, principalmente em determinados grupos étnicos, até então consideradas raras nesta faixa etária, como é caso do DM2 (ASSAO e CERVATOMANCUSO, 2008).

Dessa maneira, a relevância do estudo justifica-se pelo fato que a identificação dos principais fatores que modulam o comportamento alimentar seja imprescindível para a adaptação de teorias que possam vir a fundamentar a intervenção multiprofissional, bem como para o desenvolvimento de materiais educativos sobre alimentação direcionados para as crianças e sua família. Frente a essa realidade, torna-se de fundamental importância a promoção à saúde e prevenção de doenças crônicas não-transmissíveis (DCNT) e obesidade. Tais agravos à saúde vêm crescendo na população mundial, especialmente entre os mais jovens.

Diante do exposto, o estudo teve como objetivos analisar na literatura produções científicas acerca da relação dos hábitos alimentares para o surgimento da obesidade e do diabetes mellitus tipo 2 na infância e adolescência. 


\section{MÉTODOS}

O estudo caracteriza-se como uma revisão integrativa. Este método possibilita sumarizar as pesquisas publicadas e obter conclusões a partir da pergunta norteadora. Uma revisão integrativa bem realizada exige os mesmos padrões de rigor, clareza e replicação utilizada nos estudos primários.

A busca pelos estudos ocorreu entre os meses de fevereiro a maio de 2018. As bases de dados online pesquisadas foram: Scientific Electronic Library Online (SciELO), Literatura Latino-americana e do Caribe em Saúde (LILACS) e da Medical Literature Analysis and Retrieval System Online (MEDLINE), acessados atráves da Biblioteca Virtual em Saúde (BVS). Os descritores controlados utilizados e listados pelos descritores de Ciências da Saúde (DeCS) forão: "Alimentação", "Diabetes Mellitus", "Obesidade infantil". Os descritores foram cruzados nessa mesma ordem e por meio do operador "and".

Os critérios de inclusão foram artigos científicos que responderam aos objetivos, que estivessem na língua portuguesa e inglesa, disponíveis na íntegra e gratuitamente nas bases de dados selecionadas no período de 2008 a 2018. Optou-se pela exclusão de editais, cartas ao editor, opinião de especialistas e reflexões.

A apresentação dos resultados e da discussão dos dados obtidos foi realizada de forma descritiva, possibilitando ao leitor a avaliação da aplicabilidade da revisão integrativa elaborada, de forma a atingir o objetivo desse método. Para essa apresentação, serão elaboradas categorias temáticas, com o intuito de promover a compreensão dos conteúdos analisados.

\section{RESULTADOS}

Com as informações obtidas, elaborou-se, com os principais dados do estudo, uma tabela de acordo com o proposto na metodologia. Na tabela 1 discorre acerca das principais abordagens quanto os autores(as), título do artigo, base de dados e objetivos dos estudos

\section{DISCUSSÃO}

Após a leitura detalhada dos artigos selecionados, observou-se a necessidade de categorizá-los conforme a similaridade de conteúdo, dessa forma, foi criada duas categorias:

\section{Relação dos hábitos alimentares com a obesidade infantil e na adolescência}

A infância e adolescência apresentam como característica o crescimento físico, a alta vulnerabilidade para deficiências nutricionais e parece constituir também, período de risco para o desenvolvimento de doenças crônicas na vida adulta. Desta forma, por esse motivo tem-se priorizado a vigilância nutricional nessa fase com o objetivo de avaliar e intervir no comportamento alimentar, prevenindo assim os prejuízos ao crescimento e à saúde desse grupo (BREVIDELLI et al. 2015).

A obesidade infantil teve um aumento alarmante nas últimas décadas. Essa epidemia mundial está associada a vários fatores e é considerada um problema de saúde pública podendo desencadear diversos danos saúde das crianças, podendo citar o excesso de gordura na região abdominal, o aumento do colesterol, que são desencadeadores das doenças crônico-degenerativas (PINTO e OLIVEIRA, 2009).

Todavia, deve-se enfatizar que na adolescência ocorrem várias mudanças, tanto mundanças fisiológicas no corpo, bem como importantes mudanças psicossociais e estas mudanças têm contribuído para a vulnerabilidade ao desenvolvimento de doenças crônicas como o diabetes devido à inadequação da alimentação. Ressalta-se que na adolescência além da necessidade de uma alimentação saudável, deve-se atentar as necessidades energéticas e de nutrientes para atender à demanda do crescimento nessa fase (ENES e SLATER, 2010).

Os dados epidemiológicos da literatura apontam que na infância e adolescência, o número de obesos tem crescido elevadamente, e na maioria dos casos é resultado ao estilo de vida. Para Marchi-Alves et al. (2011) as causas endócrinas são responsáveis pela obesidade, mas caracterizam-se em menos de 10\% dos casos, enquanto que os fatores ambientais, como estilo de vida sedentário e hábitos alimentares inadequados, são determinantes neste processo. 
Tabela 1 - Caracterização dos estudos científicos de acordo com as variáveis: número de ordem, autor(es), título do artigo, base de dados e objetivos dos estudos $(\mathrm{N}=10)$.

\begin{tabular}{|c|c|c|c|c|}
\hline № & Autores & Título & $\begin{array}{c}\text { Base de } \\
\text { dados }\end{array}$ & Objetivos \\
\hline 1 & $\begin{array}{l}\text { Pinto; } \\
\text { Oliveira } \\
2009\end{array}$ & $\begin{array}{l}\text { Ocorrência de obesidade } \\
\text { infantil em pré- escolares } \\
\text { de uma creche de São } \\
\text { Paulo }\end{array}$ & SciELO & $\begin{array}{l}\text { Identificar a ocorrência de sobrepeso e } \\
\text { obesidade em crianças pré-escolares } \\
\text { (dois a cinco anos), em uma creche do } \\
\text { município de São Paulo, por meio da } \\
\text { relação peso/estatura. }\end{array}$ \\
\hline II & $\begin{array}{c}\text { Enes; Slater } \\
2010\end{array}$ & $\begin{array}{l}\text { Obesidade na } \\
\text { adolescência e seus } \\
\text { principais fatores } \\
\text { determinantes }\end{array}$ & SciELO & $\begin{array}{l}\text { Discutir os principais fatores ambientais } \\
\text { determinantes do sobrepeso e da } \\
\text { obesidade em adolescentes. }\end{array}$ \\
\hline III & $\begin{array}{c}\text { Costa et al. } \\
2011\end{array}$ & $\begin{array}{l}\text { Promoção da saúde e } \\
\text { diabetes: discutindo a } \\
\text { adesão e a motivação de } \\
\text { indivíduos diabéticos } \\
\text { participantes de } \\
\text { programas de saúde }\end{array}$ & SciELO & $\begin{array}{l}\text { Analisar, por meio de revisão } \\
\text { sistemática, informações publicadas } \\
\text { sobre a adesão e a motivação de } \\
\text { pessoas portadoras de diabetes mellitus } \\
\text { (DM) participantes de programas de } \\
\text { saúde que visam ao estímulo à adoção } \\
\text { de um estilo de vida saudável. }\end{array}$ \\
\hline IV & $\begin{array}{c}\text { Marchi-Alves } \\
\text { et al. } 2011\end{array}$ & $\begin{array}{l}\text { Obesidade infantil ontem e } \\
\text { hoje: importância da } \\
\text { avaliação antropométrica } \\
\text { pelo enfermeiro }\end{array}$ & SciELO & $\begin{array}{l}\text { Determinar a classificação nutricional } \\
\text { infantil e comparar os índices de } \\
\text { sobrepeso e obesidade de crianças } \\
\text { atendidas em uma Unidade de Saúde de } \\
\text { um município do interior paulista nos } \\
\text { anos de } 1983 / 1984 \text { e } 2003 / 2004 \text {. }\end{array}$ \\
\hline V & $\begin{array}{l}\text { Copeland et } \\
\text { al. } \\
2013\end{array}$ & $\begin{array}{l}\text { Management of Newly } \\
\text { Diagnosed Type } 2 \\
\text { Diabetes Mellitus (T2DM) } \\
\text { in Children and } \\
\text { Adolescents }\end{array}$ & MEDLINE & $\begin{array}{l}\text { Classificar da qualidade das evidências } \\
\text { e força de recomendação do diabetes } \\
\text { mellitus em crianças e adolescentes. }\end{array}$ \\
\hline VI & $\begin{array}{l}\text { Gonçalves et } \\
\text { al. } \\
2014\end{array}$ & $\begin{array}{l}\text { Análise dos materiais } \\
\text { educativos sobre diabetes } \\
\text { para crianças }\end{array}$ & MEDLINE & $\begin{array}{l}\text { Quantificar e avaliar os materiais } \\
\text { educativos/ informativos direcionados } \\
\text { para crianças com diabetes. }\end{array}$ \\
\hline VII & $\begin{array}{l}\text { Brevidelli et } \\
\text { al. } 2015\end{array}$ & $\begin{array}{l}\text { Prevalência e fatores } \\
\text { associados ao sobrepeso } \\
\text { e obesidade entre } \\
\text { adolescentes de uma } \\
\text { escola pública }\end{array}$ & LILACS & $\begin{array}{l}\text { Verificar a prevalência e os fatores } \\
\text { associados ao sobrepeso e à obesidade } \\
\text { entre adolescentes de uma escola } \\
\text { pública em Campinas, São Paulo. }\end{array}$ \\
\hline $\begin{array}{l}\text { VII } \\
\text { I }\end{array}$ & $\begin{array}{l}\text { Villa et al. } \\
\quad 2015\end{array}$ & $\begin{array}{l}\text { Padrões alimentares de } \\
\text { criancas e determinantes } \\
\text { socioeconômicos, } \\
\text { comportamentais e } \\
\text { maternos }\end{array}$ & SciELO & $\begin{array}{l}\text { Identificar os padrões alimentares de } \\
\text { crianças e verificar sua associação com } \\
\text { determinantes socioeconômicos, } \\
\text { comportamentais e maternos. }\end{array}$ \\
\hline IX & $\begin{array}{l}\text { Cunha; } \\
\text { Chiarelli; } \\
\text { Vargas } \\
2016\end{array}$ & $\begin{array}{l}\text { Síndrome metabólica em } \\
\text { crianças e adolescentes } \\
\text { com excesso de peso } \\
\text { assistidas em policlínica } \\
\text { universitária de nível } \\
\text { secundário }\end{array}$ & LILACS & $\begin{array}{l}\text { Avaliar a prevalência de síndrome } \\
\text { metabólica em crianças e adolescentes } \\
\text { com excesso de peso. }\end{array}$ \\
\hline$X$ & $\begin{array}{l}\text { Corrêa et al. } \\
\quad 2017\end{array}$ & $\begin{array}{l}\text { Padrões alimentares de } \\
\text { escolares: existem } \\
\text { diferenças entre crianças } \\
\text { e adolescentes? }\end{array}$ & SciELO & $\begin{array}{l}\text { Identificar padrões alimentares de } \\
\text { crianças e adolescentes de escolas } \\
\text { públicas e verificar a associação destes } \\
\text { com a faixa etária, o sexo, o município } \\
\text { de residência e a classe econômica. }\end{array}$ \\
\hline
\end{tabular}

Fonte: Biblioteca Virtual em Saúde. 
Neste sentido, verifica-se que a prevalência de obesidade na infância e adolescência no Brasil, resulta a uma grande parte de a população ter acesso apenas a alimentações ricas em açucares e gorduras. Além disso, essa fase da vida, os mesmos não se preocupam com as consequências da má alimentação.

Devido aos prejuízos nutricionais do adolescente ser uma temática que hoje é bastante discutida na atenção primária, é comum a presença de obesidade nesta faixa etária tem sido associada ao aparecimento precoce de várias doenças, tais como, a hipertensão arterial, a dislipidemias, o aumento da ocorrência de diabetes tipo 2, distúrbios na esfera emocional, além de comprometer a postura e causar alterações no aparelho locomotor (ENES e SLATER, 2010).

Pode-se citar, que adolescentes com idade de 10 a 19 anos tem como principais fatores de risco cardiovascular o tabagismo, o declínio na prática de atividade física, a presença de doenças crônicas em familiares, bem como o elevado consumo de alimentos que provocam doenças cardiovasculares (DCV). Aponta-se que a alimentação do adolescente apresenta alto consumo de alimentos com elevado teor de gorduras saturadas, açúcares, sal e carboidratos simples, associados à baixa ingestão de frutas, verduras e legumes (BREVIDELLI et al., 2015).

Em uma pesquisa multiétnica com crianças 6 e 4 anos apontou que práticas maternas restritivas se associaram com menor densidade energética auto selecionada pelas crianças. Neste sentido, o estudo de Villa et al. (2015) corrobora que quando a alimentação saudável é realizada pelos pais e responsáveis da criança, torna-se mais fácil a criança gostar, tendo os pais como exemplo. Dessa maneira, essa prática pode ser considerada positiva, desde que sua aplicação não tenha conotação negativa quanto ao consumo de determinados alimentos pela criança e não estimule o consumo alimentar compensatório de alimentos altamente calóricos posteriormente ou em momentos liberados pelos pais.

Sabe-se que no Brasil as pessoas gostam de segui o padrão alimentar ocidental, consumindo alimentos riscos e sal e açucares. Assim, conforme Corrêa et al. (2017) o padrão alimentar dos brasileiros é consumir salgadinhos, bolachas, doces e refrigerante, além de alimentos fritos, onde os adolescentes sofre influência no ambiente social, contribuindo assim, para interferir nos hábitos alimentares e, portanto, nesta fase tendem a ocorrer mudanças no comportamento.

\section{Diabetes mellitus tipo 2 na infância e adolescência}

De acordo com Copelande $t$ al. (2013) nas últimas três décadas, a prevalência da obesidade infantil aumentou dramaticamente na América do Norte, dando início a uma variedade de problemas de saúde, incluindo o diabetes mellitus tipo 2 (DM2), que antes não era visto até muito mais tarde na vida. $O$ rápido surgimento do DM2 na infância representa um desafio para muitos médicos, que geralmente se encontram mal equipados para tratar doenças de adultos encontradas em crianças.

Neste sentido Gonçalves et al. (2014) definem o diabetes como uma doença metabólica cuja principal característica é aumento de glicose no sangue, devido à produção alterada de insulina pelo sangue e resistência à ação do hormônio, onde também a diabetes é uma doença crônico-degenerativa de grande prevalência na população mundial configurando-se assim um sério problema de saúde pública,

Costa et al. (2011) afirma que o tratamento do DM é complexo e difícil de ser realizado, onde tem aumentado dificuldades no controle da doença. Mudanças nos hábitos de vida relacionados ao tipo de dieta ingerida, à realização de atividade física, monitoramento da glicemia, uso diário de medicamentos e de insulina constituem os fundamentos da terapia, para que se tenha uma boa qualidade de vida.

Neste sentido, observa-se o risco de complicações micro e macrovasculares em crianças e adolescentes é extremamente elevado pela precoce faixa etária do diagnóstico, levando, em longo prazo, a um imenso impacto econômico, com grande ônus ao sistema de saúde, à sociedade (CORRÊA et al. 2017).

Cada componente da Síndrome Metabólica está associado a um risco cardiovascular, a obesidade está entre os principais fatores de risco para desenvolvimento de doenças cardiovasculares. No estudo de Brevidelli et al. (2015) observou-se que 100\% dos pacientes com SM e 94,23\% dos pacientes sem SM apresentaram risco cardiovascular (RCV). No grupo com SM, 95\% deles tinham dois ou mais fatores de risco e $5 \%$ tinham somente um. $O$ fator de risco encontrado com maior frequência foi à dislipidemia. 
Tanto os casos de excesso de peso quanto de DM2 em adolescentes trazem preocupação no âmbito da saúde pública, à medida que existe, em ambas as situações, a tendência da permanência no perfil de risco durante a vida adulta. $\mathrm{O}$ acúmulo excessivo da gordura corporal, e principalmente da gordura abdominal, está associado aos valores aumentados de ácidos graxos livres ( $A G L)$ na corrente sanguínea, os quais, por sua vez, podem prejudicar a sinalização da insulina diminuindo a sensibilidade dos receptores nas membranas celulares, criando o quadro de resistência à insulina (RI) (CUNHA et al., 2016).

O impacto pela mudança alimentar no cenário familiar vem em decorrência das necessidades ou demandas para controle da doença, já que os estudos apontam que a terapia nutricional é parte imprescindível no tratamento do diabetes mellitus. Todavia, a reestruturação alimentar é uma das mudanças mais difíceis que a família tem que enfrentar no convívio com diabetes, por acarretar restrições e limitações até então desnecessárias (BREVIDELLI et al., 2015).

\section{CONSIDERAÇÕES FINAIS}

Diante do exposto, fica evidenciado que o bem-estar e a qualidade de vida relacionada com a saúde da criança e do adolescente devem ser considerados sob uma perspectiva ecológica focando vários níveis de análise, a criança, os pais e a família. A literatura mostra que o consumo das crianças e adolescentes está desequilibrado e essa realidade são fatores precursores do aumento das doenças crônicas como o diabetes mellitus nesta fase precoce que é a adolescência, acarretando ainda problema de saúde coletiva no futuro.

Neste ensejo, torna-se necessário alertar a população dos riscos nutricionais que podem surgir durante a infância e adolescência, sendo de grande importância a reeducação alimentar e a implementação de atividades de educação nutricional nas escolas, as quais podem auxiliar na formação de hábitos alimentares saudáveis, na manutenção da saúde e controle de peso corporal adequado. Salienta-se que o aumento de peso nessa idade associado com a inadequação alimentar pode contribuir para o desenvolvimento de doenças crônicas na idade adulta, que são doenças onde o estilo de vida e os hábitos inadequados de alimentação são os principais fatores de prevenção.

As informações apresentadas neste estudo devem fomentar novas pesquisas em torno do consumo alimentar e sua relação com o desencadeamento do diabetes mellitus a nível de Brasil, além de promover o diagnóstico precoce do desequilíbrio dietético nessa população jovem como medida preditora da saúde infanto-juvenil e instrumento preventivo de agravos a saúde.

\section{REFERÊNCIAS}

1. ALBIERO KA, ALVES FS. Formação e desenvolvimento de hábitos alimentares em crianças pela educação nutricional. Revista Nutrição em Pauta, $2007 ; 15(82): 17-21$

2. ASSAO TY, CERVATO-MANCUSO AMC. Alimentação Saudável: percepções dos educadores de instituições infantis. RevBras Crescimento Desenvol Hum, 2008; 18(2):126-134.

3. BRASIL. Instituto Nacional do Câncer. Inquérito domiciliar: comportamentos de risco e morbidade referida de doenças e agravos não transmissíveis. Brasília, 2009.

4. BREVIDELLI MM, COUTINHO RMC, COSTA LFV et al. Prevalência e fatores associados ao sobrepeso e obesidade entre adolescentes de uma escola pública. RevBrasPromoç Saúde, 2015; 28(3): 379-386.

5. COPELAND KC, SILVERSTEIN J, MOORE KR et al. Management of Newly Diagnosed Type 2 Diabetes Mellitus (T2DM) in Children and Adolescents. Pediatrics, 2013; 131(2): 364-382.

6. CORREA RS, VENCATO PH, ROCKETT FC et al. Padrões alimentares de escolares: existem diferenças entre crianças e adolescentes? Ciência \& Saúde Coletiva, 2017; 22(2):553-562.

7. COSTA JÁ, BALGA RSM, ALFENAS RCG et al. Promoção da saúde e diabetes: discutindo a adesão e a motivação de indivíduos diabéticos participantes de programas de saúde. Ciência \& Saúde Coletiva, 2011; 16(3): 2001-2009.

8. CUNHA JB, CHIARELLI G, VARGAS DM. Síndrome metabólica em crianças e adolescentes com excesso de peso assistidas em policlínica universitária de nível secundário. Revista da AMRIGS, 2016; 60(3): 206-213.

9. ENES CC, SLATER B. Obesidade na adolescência e seus principais fatores determinantes. RevBrasEpidemiol, 2010; 13(1):163-71.

10. GONÇALVES VM, PACÍFICO CF, TEIXEIRA PG et al. Análise dos materiais educativos sobre diabetes para crianças. Perspectivas em Psicologia, $2014 ; 18(1): 44-56$.

11. MARCHI-ALVES LM, YAGUI CM, RODRIGUES CS et al. Obesidade infantil ontem e hoje: importância da avaliação antropométrica pelo enfermeiro. Esc Anna Nery (impr.), 2011; 15(2):238-244.

12. MÜLLER RM, TOMASI E, FACCHINI LA et al. Excesso de peso e fatores associados em menores de cinco anos em populações urbanas no Brasil. RevBrasEpidemiol, 2014; 285-296.

13. NEUTZLING MG, ASSUNÇÃO MCF, MALCON MC et al. Hábitos alimentares de escolares adolescentes de Pelotas, Brasil. Rev. Nutr 2010; 23(3):379-388.

14. PINTO MCM, OLIVEIRA AC. Ocorrência de obesidade infantil em pré- escolares de uma creche de São Paulo. Einstein, 2009; 7(2):170-175.

15. VILLA JKD, SILVA AR, SANTOS TSS et al. Padrões alimentares de crianças e determinantes socioeconômicos, comportamentais e maternos. Rev Paul Pediatr, 2015; 33(3):302-309. 